\title{
PREDICTION OF CARCASS TRAITS IN FATTENING FRIESIAN CROSSBRED AND BUFFALO MALE CALVES BY USING BODY MEASUREMENTS
}

\author{
Dalia K.A. EL-Hedainy ${ }^{1}$, M.G.A Latif and A.E. Mahdy \\ Department of Animal Production, Faculty of Agriculture, Alexandria University, Egypt \\ ${ }^{I}$ corresponding auther.e-mail:dheddany@yahoo.com and drdalia.dh@gmail.com
}

\section{SUMMARY}

Five body measurements were taken on 142 Friesian crossbred and 49 buffalo male calves. 14 Friesian crossbred and 12 buffalo male calves slaughtered at 435+17.5 $\mathrm{kg}$ average body weight and at about 15-17 months of age. The aims of the present study were to estimate the relationship between body measurements and slaughter weight, and carcass characteristics. Body measurements were used to predict carcass traits.

The slaughter weight was positively and significantly correlated with most of carcass traits in range from 0.15 to 0.98 and 0.13 to 0.89 for both Friesian crossbred and buffalo carcasses, respectively. Both of slaughter weight and heart girth showed high accuracy ranged from 0.33 to 0.97. Round circumference (RC) and body length (BL) had highly accuracy for predicting most of carcass traits in both Friesian crossbred and buffalo carcass traits with values of 0.89 to 0.99 , respectively. The present results concluded that, the heart girth $(H G)$ of Frisian crossbred calves and each of round circumference (RC) and body length (BL) of buffalo calves were the best indicators to predict most of their carcass traits.

\section{Keywords: prediction, measurements, carcass, Friesian crossbred, buffalo}

\section{INTRODUCTION}

Animal body conformation in cattle is one of the important bases to judge and select animals for breeding or production purposes. Measurements of some parts of the animal body give an objective description of the animal body conformation. The ability of cattle to produce meat may be estimated with these measurements if relationships between body measurements and meat production characters were strongYao et al., 1953.

Various studies have been made on the relationships of body measurements to some of the performance factors of beef cattle, such as Kohliet al. (1951) found relationships among five body measurements to slaughter grade, carcass grade and dressing percentage. Gilbert et al. (1993) studied the relation between body dimensions and carcass measurements of cattle selected for post weaning gain. Afolayan et al. (2002) predicted carcass composition in cross cattle by using live animal measurements.

The objective of the present investigation wa sto determine, the relationship between each of body measurements and slaughter weight and carcass characteristics. Prediction of carcass traits using body measurements was also studied.

\section{MATERIALS AND METHODS}

Data on body weight and body measurements for 191Friesian crossbred and buffalo male calves were taken from the herd belonging to the Rations and Fattening Unit, Faculty of Agriculture, Alexandria University. All calves were bought from local markets in Delta and their average initial weights were $195 \pm 3.61 \mathrm{~kg}$ and their average estimated ages were about 9-11 months (it was measured by teething method). The calves were fattened for 6 months under the same management and environmental conditions. Animals were housed free in open yards and fed commercial mixture concentrate ration (14\% $\mathrm{TP}$ and $65 \%$ TDN) according to their body weights ( $2 \%$ of body weight) (NRC, National Research Council, 2001). Also, chopped wheat straw (at the rate of $1 \%$ of body weight) and green forage (at rate of $5 \mathrm{~kg} / \mathrm{head} /$ day) were offered besides concentrate diet.

Body measurements were recorded before slaughter as described by Fisher (1976) including height at withers (HW), body length (BL), diagonal length (DL), heart girth (HG) and circumference of round (CR). Slaughter weights (SW) of the animals were recorded immediately before the slaughter for 12 male buffalo and 14 male Friesian crossbred calves which were randomly chosen to be slaughtered. Their average weights were $435 \pm 17.55$ $\mathrm{kg}$, and their estimated ages were 15-17 months (it was measured by teething method). The calves were fasted for 14 hours before slaughtering. The animals were slaughtered by severing the jugular vein and after complete bleeding; they were skinned and dressed out. Hot carcass weight (HCW) was recorded before carcasses were chilled for 24 hours at $5^{\circ} \mathrm{C}$. Cold carcass weight (CCW) was recorded and then the carcasses were split lengthwise into two halves. Each half was weighed and then was divided into fore and hind quarters ( $\mathrm{Fq}$ and $\mathrm{Hq}$, respectively). The separation of the quarters was at the $13^{\text {th }}$ rib. Quarters were dissected into boneless meat (muscles and fat) and bone. The excess fat was separated from boneless meat and was weighed. Weights of boneless meat (BM) and bone were recorded. Boneless meat and dressing percentages were calculated as follows: Boneless meat $(\mathrm{BM}) \%=(\mathrm{BM} / \mathrm{HCW}) \times 100$ Dressing percentage $(\mathrm{DR} \%)=\mathrm{HCW}$ or $\mathrm{CCW} / \mathrm{SWx} 100$. 
Weights of some high price cuts (prime cuts) i.e. Fillet (Longissimusdorsimuscle), Telbianco (Semitendenosusmuscle) and Roast beef (Semimembranosus)were recorded.

The data were statistically analyzed according to statistical analysis system (SAS, 2004). The following models were assumed to describe the observation in the two studies:

Where:

$$
Y i j k=\mu+b j(x-x-)+e i j k
$$

Yijk = observation of each trait studied (body weight, body measurements and carcass traits),

$\mu=$ the overall mean,

$\mathrm{bj}=\mathrm{a}$ regression coefficient of the trait on body measure $(\mathrm{BM})$ or slaughter weight $(\mathrm{SW})$,

$\mathrm{x}=$ the independent continues variate for $(\mathrm{SW})$ or (BM),

$\mathrm{x}-=$ the respective mean and,

eijk $=$ the random error $(0, \delta 2)$

Simple correlation and regression coefficients between slaughter weight and body measurements and carcass characteristics were calculated. Besides, stepwise multi regressions of carcass traits on slaughter weight and body measurements were calculated to get the best equations to predict the carcass traits. The following models were assumed to describe the observation in the two studies:

\section{RESULTS AND DISCUSSION}

\section{Simple correlation and regression coefficient:}

The descriptive statistics of the two species are presented in Tables (1 and 2). Simple correlation coefficients between both of slaughter weight and body measurements at slaughter with carcass traits of Friesian crossbred male calves are presented in Table (3). The slaughter weight was positively and significantly correlated with most carcass traits. This is agreement with, that reported by Shelton et al. (1977); Rahman (2007) and Bonvillani et al. (2010). The positive correlation coefficients ranged from 0.15 to 0.98 , meaning that when SW increases, most carcass traits tend to increase.

Most of correlations between HW and carcass traits were positive and all of them were nonsignificant except those with $\mathrm{Hq}$ weight (hot and cold) and bone. All correlations between BL and carcass traits were positive except that with Roast beef. The DL was strongly and highly significantly correlated with Telbianco and moderate, negative and significant with DR\% 9 hot and cold). The heart girth (HG) was negatively and significantly correlated with most of carcass traits. In general, the round circumference (RC) was low and non-significantly correlated with all carcass traits, except between RC and CCW (-0.60).

Variables like height, length, girth, stifle, hip and measure of muscularity (Afolayan et al., 2001) which are directly related to size and weight, displayed moderately to high positive correlations with quantity carcass components in rang $(0.27-0.94)$. This is close to that found in the present study, the measures
SW, HW, BL, DL, HG and RC were correlated with carcass components in range (0.08 - 0.83) of Frisian crossbred and (0.38 -0.99) in buffalo.

Simple regression coefficients of different carcass traits under this study on slaughter weight and body measurements at the slaughter for Friesian crossbred male calves were calculated (Table 3). Both of slaughter weight and heart girth (HG) showed higher accuracy $\left(\mathrm{R}^{2} \%\right)$ which ranged from 0.33 to 0.97 , to predict hot carcass weight, fore quarters weight (hot and cold), hind quarters weight (hot and cold), bone, meat and fat weights while, diagonal length (DL) was the best measure to predict dressing percentages.

The relationships between both slaughter weight and body measurements at slaughter with carcass traits of buffalo male calves are shown in Table (4). Similarly, slaughter weight was positively and significantly correlated with most of carcass traits and their correlation coefficients ranged from 0.13 to 0.89 . HW had highly significant correlations with some carcass traits, these were: $\mathrm{HCW}, \mathrm{CCW}$, Hqw (hot and cold), bone, boneless meat $\%$, fat and Roast beef. The BL had significant correlations (negative or positive) with most of carcass traits $\mathrm{HCW}, \mathrm{CCW}$, DR\% (hot and cold), Fqw hot, Hqw hot, meat, boneless \%, fat, Telbianco, Fillet and Roast beef. The significant correlations (negative or positive) were showed between DL and most of carcass traits. They were $\mathrm{HCW}, \mathrm{CCW}, \mathrm{DR} \%$ (hot and cold), Hqw (hot and cold), Fqw cold, bone, meat, boneless meat \%, fat, Telbianco, Fillet and Roast beef. The highly significant correlations were found between $\mathrm{HG}$ and each of $\mathrm{HCW}, \mathrm{CCW}$, Hqw (hot and cold), bone, boneless meat $\%$, fat and Roast beef. The coefficients of correlation between RC and all carcass traits under study were strong, positive or negative and significant; except that with Fqw (hot) (0.45) was moderate and non- significant.

From these results, the RC is considered the first body measure which positively and strongly associated with most carcass traits of buffalo male calves under study and followed by BL, DL or HG and therefore, they are most important indicators to predict the respective carcass traits. The simple regression coefficients shown in table (2), emphasis that, the best body measure to predict most of carcass traits of buffalo male calves is $\mathrm{RC}$ with higher accuracy ( $\mathrm{R}^{2} \%$ ) being from 0.46 to 0.98 .

Ayyatet al. (1997) estimated the correlation coefficients between live measurements and carcass components of Egyptian cattle calves slaughtered at $406 \mathrm{~kg}$ and they found that, SW was positively and significantly correlated with carcass weight and boneless meat weight ( $\mathrm{r}=0.97$ and 0.91 , respectively). Also, heart girth was significantly correlated with the same traits $(\mathrm{r}=0.75$ and 0.67 , respectively).

Rashad (2009) studied carcass traits of fattening male buffalo calves and its relationship with live body weight and body measurements, and found that, all correlation coefficients between carcass traits and body weights or body measurements were positive, higher and significant or highly significant except 
those between width at pin bones and each of boneless meat, carcass bones and fat were not significant and being 52, 55 and 53\%, respectively. The previous results were in agreement with those found in the present study.

\section{Stepwise regression analysis of carcass traits on different body measurements:}

Stepwise multiple regression analysis of carcass traits on slaughter weight and body measurements of Friesian crossbred male calves is shown in Table (5). Heart girth $(\mathrm{HG})$ was the first independent variable included in stepwise regression equation followed by SW to predict HCW with higher accuracy $\left(\mathrm{R}^{2}=0.94\right)$ and the other body measurements did not greatly change in $\mathrm{R}^{2}$ value. $\mathrm{HG}, \mathrm{SW}$ and $\mathrm{HW}$ were considered the best indicators to predict DR \% (hot or cold) with higher accuracy $\left(\mathrm{R}^{2}=0.91\right)$.

Only HG was the most effective indicator to predict both of Fqw hot and cold with higher accuracy (0.96 and 0.95 , respectively). SW followed by HW was considered the best ones to predict both Hqw hot and cold with higher accuracy (0.96 and 0.94 , respectively). CCW could be predicted with highly accuracy (0.95) on basis of HG, HW and SW. Both of SW and BL were used to predict bone weight with higher accuracy (0.99). Only SW was the best indicator to predict meat weight with higher accuracy (0.99). Besides, SW and HG followed by DL were the most effective indicators to predict boneless meat $\%$ with highly accuracy (0.99).

Fat could be predicted with the highest accuracy (0.99) by using each of SW, DL and HG. Each of Telbianco and Roast beef were depended on both SW and $\mathrm{HG}$ with accuracy of 0.88 with RC at accuracy of 0.98, respectively. However, SW and HW followed by $\mathrm{BL}$ were the most effective indicators to predict Fillet with higher accuracy of 0.97 as shown in Table (3). These results are in agreement with that found by Afolayan et al. (2002) when studied live measurements of weight, height, length, girth, fat depth, stifle- and hip-width were obtained prior to slaughter to develop prediction equations for carcass traits and recorded that, The live-weight was the most accurate predictor of carcass quantity components [meat $\left(\mathrm{R}^{2}=0.70\right)$ and bone $\left(\mathrm{R}^{2}=0.62\right)$ weight $]$.

Table 1. Least square means $( \pm$ S.E) of body measurements $(\mathrm{cm}$.$) of fattening Friesian crossbred and$ buffalo male caves

\begin{tabular}{lcc}
\hline \multirow{2}{*}{ Measures } & \multicolumn{2}{c}{ Genotype } \\
\cline { 2 - 3 } & Friesian crossbred & buffalo \\
\hline No. of calves & 142 & 49 \\
$\mathrm{HW}^{1}$ & $123.2 \pm 0.23$ & $131.7 \pm 0.54$ \\
$\mathrm{BL}^{2}$ & $87.8 \pm 0.37$ & $78.9 \pm 0.62$ \\
$\mathrm{DL}^{3}$ & $97.8 \pm 0.28$ & $94.1 \pm 0.48$ \\
$\mathrm{HG}^{4}$ & $171.7 \pm 0.45$ & $180.4 \pm 0.78$ \\
$\mathrm{RC}^{5}$ & $70.4 \pm 0.32$ & $74.6 \pm 0.61$ \\
\hline $\mathrm{HW}^{1}$, height at wither; $\mathrm{BL}^{2}$, body length; $\mathrm{DL}^{3}$ diagonal length ${ }^{4}$ HG, heart girth; $\mathrm{RC}^{5}$, Round circumference.
\end{tabular}

Table 2. Least square means $( \pm$ S.E) of slaughter weight and carcass treats of Friesian crossbred and buffalo male calves

\begin{tabular}{|c|c|c|}
\hline \multirow[b]{2}{*}{ Carcass traits } & \multicolumn{2}{|c|}{ Genotype } \\
\hline & Friesian crossbred & buffalo \\
\hline No. of carcass & 14 & 12 \\
\hline Slaughter weight & $415.85 \pm 18.83$ & $455.66 \pm 16.27$ \\
\hline $\mathrm{HCW}^{1}, \mathrm{~kg}$ & $243.03 \pm 4.39$ & $247.70 \pm 4.76$ \\
\hline $\mathrm{DR}^{2} \%$ (hot) & $58.44 \pm 4.03$ & $54.36 \pm 1.02$ \\
\hline $\mathrm{Fqw}^{3}($ hot $), \mathrm{kg}$ & $121.14 \pm 3.00$ & $119.70 \pm 3.25$ \\
\hline $\mathrm{Hqw}^{4}(\mathrm{hot}), \mathrm{kg}$ & $122.48 \pm 2.25$ & $128.16 \pm 2.43$ \\
\hline $\mathrm{CCW}^{5}, \mathrm{~kg}$ & $236.20 \pm 15.45$ & $234.80 \pm 11.74$ \\
\hline DR\%(cold) & $56.00 \pm 3.25$ & $51.53 \pm 2.47$ \\
\hline $\mathrm{Fqw}$ (cold),kg & $117.10 \pm 8.63$ & $116.56 \pm 6.06$ \\
\hline $\mathrm{Hqw}$ (cold), $\mathrm{kg}$ & $119.00 \pm 8.71$ & $118.27 \pm 6.62$ \\
\hline Bone, $\mathrm{kg}$ & $40.76 \pm 1.02$ & $43.33 \pm 1.21$ \\
\hline Meat, kg & $155.03 \pm 6.19$ & $148.14 \pm 7.35$ \\
\hline Boneless meat, \% & $65.63 \pm 1.27$ & $63.09 \pm 1.55$ \\
\hline Fat, $\mathrm{kg}$ & $20.18 \pm 1.89$ & $17.73 \pm 2.25$ \\
\hline \multicolumn{3}{|l|}{ High price cuts: } \\
\hline Telbianco $^{6}, \mathrm{~kg}$ & $4.66 \pm 0.32$ & $3.60 \pm 0.34$ \\
\hline Fillet $^{7}, \mathrm{~kg}$ & $4.58 \pm 0.24$ & $4.55 \pm 0.26$ \\
\hline Roast beef ${ }^{8}, \mathrm{~kg}$ & $11.50 \pm 0.59$ & $8.90 \pm 0.64$ \\
\hline
\end{tabular}

$\mathrm{HCW}^{1}$, hot carcass weight; $\mathrm{DR}^{2}$, dressing percentage; $\mathrm{Fqw}^{3}$; fore quarter weight; $\mathrm{Hqw}^{4}$, hind quarter weight; $\mathrm{CCW}^{5}$, cold carcass weight; Telbianco ${ }^{6}$ and Roast beef ${ }^{7}$, cuts from round; Fillet ${ }^{8}$, cut from short loin. 
Table 3. Simple regression and correlation coefficients between carcass traits(kg.) and body measurements $(\mathrm{cm}$.) for fattening Friesian crossbred male calves $(\mathrm{n}=14)$

\begin{tabular}{|c|c|c|c|c|c|c|}
\hline $\begin{array}{l}\text { Independent / } \\
\text { Dependent } \\
\text { variable }\end{array}$ & $\begin{array}{l}\mathrm{SW}^{1} \\
(\mathrm{~kg})\end{array}$ & $\begin{array}{l}\mathbf{H W}^{2} \\
\text { (cm.) }\end{array}$ & $\begin{array}{l}\mathrm{BL}^{3} \\
\text { (cm.) }\end{array}$ & $\begin{array}{c}\mathrm{DL}^{4} \\
\text { (cm.) }\end{array}$ & $\begin{array}{l}\mathrm{HG}^{5} \\
\text { (cm.) }\end{array}$ & $\begin{array}{l}\mathrm{RC}^{6} \\
\text { (cm.) }\end{array}$ \\
\hline $\mathrm{HCW}^{7}, \mathrm{~kg}$ & $\begin{array}{c}0.54[0.87] \\
\left(0.96^{* * *}\right)\end{array}$ & $\begin{array}{c}3.17[0.24] \\
\left(0.49^{\mathrm{ns}}\right)\end{array}$ & $\begin{array}{c}9.88[0.50] \\
\left(0.70^{* *}\right)\end{array}$ & $\begin{array}{c}1.18[0.06] \\
\left(0.25^{\mathrm{ns}}\right)\end{array}$ & $\begin{array}{c}-5.93[0.86] \\
\left(-0.93^{* *}\right)\end{array}$ & $\begin{array}{c}2.69[0.28] \\
\left(0.16^{\mathrm{ns}}\right)\end{array}$ \\
\hline $\mathrm{DR}^{8} \%$ (hot) & $\begin{array}{c}-0.004[0.01] \\
\left(-0.13^{\text {ns }}\right)\end{array}$ & $\begin{array}{c}0.11[0.01] \\
\left(0.11^{\mathrm{ns}}\right)\end{array}$ & $\begin{array}{c}0.04[0.00] \\
\left(0.02^{\text {ns }}\right)\end{array}$ & $\begin{array}{c}-0.44[0.03] \\
\left(-0.62^{*}\right)\end{array}$ & $\begin{array}{c}-0.29[0.49] \\
\left(-0.30^{\mathrm{ns}}\right)\end{array}$ & $\begin{array}{c}-0.26[0.12] \\
\left(-0.11^{\mathrm{ns}}\right)\end{array}$ \\
\hline $\mathrm{Fqw}^{9}$ (hot), $\mathrm{kg}$ & $\begin{array}{c}0.28[0.75] \\
\left(0.82^{* * *}\right)\end{array}$ & $\begin{array}{c}1.06[0.09] \\
\left(0.31^{\mathrm{ns}}\right)\end{array}$ & $\begin{array}{c}4.68[0.38] \\
\left(0.62^{*}\right)\end{array}$ & $\begin{array}{c}0.07[0.00] \\
\left(0.02^{\text {ns }}\right)\end{array}$ & $\begin{array}{c}-3.36[0.96] \\
\left(-.098^{* * *}\right)\end{array}$ & $\begin{array}{c}-0.13[0.00] \\
\left(-0.01^{\mathrm{ns}}\right)\end{array}$ \\
\hline $\mathrm{Hqw}^{10}$ (hot),kg & $\begin{array}{c}0.26[0.94] \\
\left(0.98^{* * *}\right)\end{array}$ & $\begin{array}{c}2.10[0.40] \\
\left(0.63^{*}\right)\end{array}$ & $\begin{array}{c}5.20[0.51] \\
\left(0.71^{* *}\right)\end{array}$ & $\begin{array}{c}1.11[0.20] \\
\left(0.45^{\mathrm{ns}}\right)\end{array}$ & $\begin{array}{c}-2.50[0.59] \\
\left(-0.77^{* *}\right)\end{array}$ & $\begin{array}{c}2.82[0.12] \\
\left(0.34^{\mathrm{ns}}\right)\end{array}$ \\
\hline $\mathrm{CCW}^{11}, \mathrm{~kg}$ & $\begin{array}{c}0.50[0.39] \\
\left(0.62^{*}\right)\end{array}$ & $\begin{array}{c}-6.09[0.23] \\
\left(-0.47^{\mathrm{ns}}\right)\end{array}$ & $\begin{array}{c}1.88[0.00] \\
\left(0.06^{\mathrm{ns}}\right)\end{array}$ & $\begin{array}{c}0.72[0.01] \\
\left(0.07^{\mathrm{ns}}\right)\end{array}$ & $\begin{array}{c}-8.11[0.39] \\
\left(-0.63^{*}\right)\end{array}$ & $\begin{array}{c}-19.47[0.37] \\
\left(-0.60^{*}\right)\end{array}$ \\
\hline DR\%(cold) & $\begin{array}{c}-0.001[0.00] \\
\left(-0.02^{\mathrm{ns}}\right)\end{array}$ & $\begin{array}{c}0.08[0.01] \\
\left(0.08^{\mathrm{ns}}\right)\end{array}$ & $\begin{array}{c}0.08[0.00] \\
\left(0.03^{\text {ns }}\right)\end{array}$ & $\begin{array}{c}-0.44[0.38] \\
\left(-0.61^{*}\right)\end{array}$ & $\begin{array}{c}-0.35[0.13] \\
\left(-0.36^{\text {ns }}\right)\end{array}$ & $\begin{array}{l}-0.35[0.02] \\
\left(-0.14^{\mathrm{ns}}\right)\end{array}$ \\
\hline $\mathrm{Fqw}$ (cold), $\mathrm{kg}$ & $\begin{array}{c}0.29[0.75] \\
\left(0.86^{* *}\right)\end{array}$ & $\begin{array}{c}0.97[0.09] \\
\left(0.29^{\mathrm{ns}}\right)\end{array}$ & $\begin{array}{c}4.38[0.36] \\
\left(0.59^{*}\right)\end{array}$ & $\begin{array}{c}0.06[0.00] \\
\left(0.02^{\mathrm{ns}}\right)\end{array}$ & $\begin{array}{c}-3.27[0.95] \\
\left(-0.97^{* * * *}\right)\end{array}$ & $\begin{array}{l}-0.34[0.00] \\
\left(-0.04^{\mathrm{ns}}\right)\end{array}$ \\
\hline $\mathrm{Hqw}$ (cold), $\mathrm{kg}$ & $\begin{array}{c}0.24[0.93] \\
\left(0.96^{* * *}\right)\end{array}$ & $\begin{array}{c}2.01[0.36] \\
\left(0.60^{*}\right)\end{array}$ & $\begin{array}{c}5.37[0.54] \\
\left(0.73^{* *}\right)\end{array}$ & $\begin{array}{c}1.04[0.18] \\
\left(0.41^{\mathrm{ns}}\right)\end{array}$ & $\begin{array}{c}-2.74[0.67] \\
\left(-0.81^{* *}\right)\end{array}$ & $\begin{array}{c}2.60[0.09] \\
\left(0.00^{\text {ns }}\right)\end{array}$ \\
\hline Bone,kg & $\begin{array}{c}0.09[0.96] \\
\left(0.50^{\mathrm{ns}}\right)\end{array}$ & $\begin{array}{c}0.64[0.32] \\
\left(0.57^{*}\right)\end{array}$ & $\begin{array}{c}2.06[0.69] \\
\left(0.83^{* *}\right)\end{array}$ & $\begin{array}{c}0.38[0.21] \\
\left(0.45^{\mathrm{ns}}\right)\end{array}$ & $\begin{array}{c}-0.82[0.54] \\
\left(-0.73^{* *}\right)\end{array}$ & $\begin{array}{c}1.0[0.14] \\
\left(0.37^{\mathrm{ns}}\right)\end{array}$ \\
\hline Meat, kg & $\begin{array}{c}0.46[0.97] \\
\left(0.24^{\mathrm{ns}}\right)\end{array}$ & $\begin{array}{c}2.74[0.18] \\
\left(0.42^{\mathrm{ns}}\right)\end{array}$ & $\begin{array}{c}9.16[0.42] \\
\left(0.65^{*}\right)\end{array}$ & $\begin{array}{c}2.32[0.24] \\
\left(0.48^{\mathrm{ns}}\right)\end{array}$ & $\begin{array}{c}-4.27[0.44] \\
\left(-0.66^{*}\right)\end{array}$ & $\begin{array}{c}2.78[0.03] \\
\left(0.17^{\mathrm{ns}}\right)\end{array}$ \\
\hline Boneless meat \% & $\begin{array}{c}0.02[0.44] \\
\left(-0.36^{\mathrm{ns}}\right)\end{array}$ & $\begin{array}{c}0.19[0.10] \\
\left(0.31^{\mathrm{ns}}\right)\end{array}$ & $\begin{array}{c}0.55[0.17] \\
\left(0.41^{\mathrm{ns}}\right)\end{array}$ & $\begin{array}{c}0.06[0.02] \\
\left(0.14^{\mathrm{ns}}\right)\end{array}$ & $\begin{array}{c}-0.12[0.04] \\
\left(-0.20^{\text {ns }}\right)\end{array}$ & $\begin{array}{c}-0.12[0.01] \\
\left(-0.08^{\mathrm{ns}}\right)\end{array}$ \\
\hline Fat,kg & $\begin{array}{l}0.09[0.64] \\
\left(0.55^{*}\right)\end{array}$ & $\begin{array}{c}0.77[0.14] \\
\left(0.37^{\mathrm{ns}}\right)\end{array}$ & $\begin{array}{c}2.88[0.41] \\
\left(0.63^{*}\right)\end{array}$ & $\begin{array}{l}0.47[0.09] \\
\left(0.31^{\mathrm{ns}}\right)\end{array}$ & $\begin{array}{c}-0.21[0.34] \\
\left(-0.58^{*}\right)\end{array}$ & $\begin{array}{l}0.21[0.00] \\
\left(0.04^{\text {ns }}\right)\end{array}$ \\
\hline$\frac{\text { Prime cuts: }}{\text { Telbianco }^{12}, \mathrm{~kg}}$ & $\begin{array}{c}0.01[0.68] \\
\left(0.66^{* *}\right)\end{array}$ & $\begin{array}{c}0.03[0.04] \\
\left(0.18^{\mathrm{ns}}\right)\end{array}$ & $\begin{array}{c}0.14[0.10] \\
\left(0.32^{\text {ns }}\right)\end{array}$ & $\begin{array}{c}0.10[0.49] \\
\left(0.70^{* *}\right)\end{array}$ & $\begin{array}{c}0.01[0.00] \\
\left(0.00^{\mathrm{ns}}\right)\end{array}$ & $\begin{array}{c}0.15[0.09] \\
\left(0.31^{\mathrm{ns}}\right)\end{array}$ \\
\hline Fillet $^{13}, \mathrm{~kg}$ & $\begin{array}{c}0.005[0.65] \\
\left(0.57^{*}\right)\end{array}$ & $\begin{array}{c}-0.002[0.00] \\
\left(-0.02^{\mathrm{ns}}\right)\end{array}$ & $\begin{array}{c}0.13[0.36] \\
\left(0.59^{*}\right)\end{array}$ & $\begin{array}{c}0.02[0.08] \\
\left(0.28^{\mathrm{ns}}\right)\end{array}$ & $\begin{array}{c}-0.06[0.44] \\
\left(-0.66^{*}\right)\end{array}$ & $\begin{array}{c}-0.03[0.02] \\
\left(-0.13^{\mathrm{ns}}\right)\end{array}$ \\
\hline Roast beef ${ }^{14}, \mathrm{~kg}$ & $\begin{array}{c}0.03[0.59] \\
\left(0.15^{\mathrm{ns}}\right) \\
\end{array}$ & $\begin{array}{c}-0.04[0.0 .2] \\
\left(-0.12^{\mathrm{ns}}\right) \\
\end{array}$ & $\begin{array}{c}-0.11[0.02] \\
\left(-0.13^{\mathrm{ns}}\right)\end{array}$ & $\begin{array}{c}0.07[0.01] \\
\left(0.30^{\mathrm{ns}}\right) \\
\end{array}$ & $\begin{array}{c}0.09[0.07] \\
\left(0.27^{\mathrm{ns}}\right) \\
\end{array}$ & $\begin{array}{c}-0.22[0.07] \\
\left(-0.25^{\mathrm{ns}}\right) \\
\end{array}$ \\
\hline
\end{tabular}

[ ] Accuracy of simple regression, $\mathrm{R}^{2}$. ( ) Simple correlation coefficient.

Ns: non-significant* Significant at $\mathrm{P}<0.05$. ** Significant at $\mathrm{p}<0.01$. *** Significant at $\mathrm{P}<0.001$.

$\mathrm{SW}^{1}$,slaughter weight; $\mathrm{HW}^{2}$, height at wither; $\mathrm{BL}^{3}$, body length; $\mathrm{DL}^{4}$ diagonal length ${ }^{5}$; $\mathrm{HG}$, heart girth; $\mathrm{RC}^{6}$, Round circumference; $\mathrm{HCW}^{7}$, hot carcass weight; $\mathrm{DR}^{8}$, dressing percentage; $\mathrm{Fqw}^{9}$; fore quarter weight; $\mathrm{Hqw}^{10}$, hind quarter weight; $\mathrm{CCW}^{11}$, cold carcass weight; Telbianco ${ }^{12}$ and Roast beef ${ }^{13}$, cuts from round; Fillet $^{14}$, cut from short loin.

Stepwise multiple regression analysis of carcass traits on SW and body measurements for buffalo male calves is calculated and shown in Table (6). RC is the best indicator to predict each of $\mathrm{CCW}$, Roast beef and Telbianco with higher accuracy.

Also, only BL is the most effective indicator to predict each of DR\% hot and cold, meat and Fillet weight with higher accuracy $\left(\mathrm{R}^{2} \%\right)$, which ranged from 0.90 to 0.99 .

Both of bone weight and boneless meat \% could be predicted on basis of DL as a best indicator with higher accuracy (0.99 and 0.94, respectively). Whereas, HW is considered the best indicator to predict Hqw cold with accuracy of 0.99 . Moreover, $\mathrm{BL}$ followed by $\mathrm{RC}$ were the best indicators to predict Fqw hot, and that DL followed by HG were also the best ones to predict Fqw cold with highly accuracy of 0.99 and 0.97 , respectively. This in contrast with what was found in Frisian crossbred and this is agreement with that reported by Afolayan et al. (2002). Carcass weight may be reasonably predicted based on live animal measurement regardless of the animal type. However, for precision in other carcass traits (including meat weight and percent meat in the carcass), the breed difference is still required to develop their equations. Effective and reliable prediction of carcass yields from the low-cost objective rather than high-cost objective (e.g. ultrasound) and/or subjective live animal measurements would reduce production costs and wider application by producers.

Rashad (2009) predicted carcass performance depending on live weight and measurements of Egyptian buffalo calves, and reported that, the live body weight at slaughter was the best live measurement to predict hot and cold carcass weight $\left(\mathrm{R}^{2}=97.0\right.$ and $97.4 \%$, respectively) and followed with width at pin bones and chest girth but in very low change in $\mathrm{R}^{2}$ by 1.9 or $1.8 \%$. However, chest girth was the best measurement to predict boneless meat weight and carcass fat weight $\left(\mathrm{R}^{2}=90.6\right.$, $78.2 \%$, respectively). These results are in agreement with those found in the present study. 
Table 4. Simple regression and correlation coefficients between carcass traits (kg.) and body measurements for fattening buffalo male calves $(n=12)$

\begin{tabular}{|c|c|c|c|c|c|c|c|c|}
\hline $\begin{array}{l}\text { Independent / } \\
\text { Dependent } \\
\text { variable }\end{array}$ & $\begin{array}{l}\mathrm{SW}^{1} \\
(\mathrm{~kg})\end{array}$ & $\begin{array}{l}\mathrm{HW}^{2} \\
\text { (cm.) }\end{array}$ & $\begin{array}{c}\mathrm{BL}^{3} \\
(\mathrm{~cm} .)\end{array}$ & \multicolumn{2}{|c|}{$\begin{array}{c}\mathrm{DL}^{4} \\
(\mathrm{~cm} .)\end{array}$} & $\begin{array}{l}\mathrm{HG}^{5} \\
(\mathrm{~cm} .)\end{array}$ & \multicolumn{2}{|c|}{$\begin{array}{l}\mathrm{RC}^{6} \\
(\mathrm{~cm} .)\end{array}$} \\
\hline $\mathrm{HCW}^{7}, \mathrm{~kg}$ & $\begin{array}{c}0.63[0.75] \\
\left(0.87^{* * *}\right)\end{array}$ & $\begin{array}{c}0.05[0.64] \\
\left(0.79^{* *}\right)\end{array}$ & $\begin{array}{c}-0.72[0.71] \\
\left(-0.84^{* *}\right)\end{array}$ & \multicolumn{2}{|c|}{$\begin{array}{c}-2.37[0.78] \\
\left(-0.88^{* *}\right)\end{array}$} & $\begin{array}{c}0.98[0.73] \\
\left(0.85^{* * *}\right)\end{array}$ & \multicolumn{2}{|c|}{$\begin{array}{l}1.46[0.89] \\
\left(0.94^{* *}\right)\end{array}$} \\
\hline $\mathrm{DR}^{8} \%$ (hot) & $\begin{array}{c}0.01[0.05] \\
\left(0.25^{\mathrm{ns}}\right)\end{array}$ & $\begin{array}{c}-0.01[0.13] \\
\left(-0.35^{\text {ns }}\right)\end{array}$ & $\begin{array}{c}0.21[0.98] \\
\left(0.99^{* * *}\right)\end{array}$ & \multicolumn{2}{|c|}{$\begin{array}{c}0.46[0.49] \\
\left(0.69^{*}\right)\end{array}$} & $\begin{array}{c}-0.10[0.14] \\
\left(-0.36^{\text {ns }}\right)\end{array}$ & \multicolumn{2}{|c|}{$\begin{array}{c}-0.34[0.02] \\
\left(-0.90^{* * *}\right)\end{array}$} \\
\hline $\mathrm{Fqw}^{9}(\mathrm{hot}), \mathrm{kg}$ & $\begin{array}{c}0.26[0.66] \\
\left(0.79^{* *}\right)\end{array}$ & $\begin{array}{c}-0.00[0.08] \\
\left(-0.27^{\mathrm{ns}}\right)\end{array}$ & $\begin{array}{c}-0.06[0.56] \\
\left(-0.74^{*}\right)\end{array}$ & \multicolumn{2}{|c|}{$\begin{array}{c}-0.02[0.01] \\
\left(-0.09^{\text {ns }}\right)\end{array}$} & $\begin{array}{c}-0.02[0.04] \\
\left(-0.21^{\mathrm{ns}}\right)\end{array}$ & \multicolumn{2}{|c|}{$\begin{array}{c}0.07[0.21] \\
\left(0.45^{\mathrm{ns}}\right)\end{array}$} \\
\hline $\mathrm{Hqw}^{10}$ (hot), $\mathrm{kg}$ & $\begin{array}{c}0.36[0.76] \\
\left(0.88^{* * *}\right)\end{array}$ & $\begin{array}{c}0.06[0.72] \\
\left(0.48^{\text {ns }}\right)\end{array}$ & $\begin{array}{c}-0.76[0.70] \\
\left(-0.83^{* *}\right)\end{array}$ & \multicolumn{2}{|c|}{$\begin{array}{c}-2.74[0.91] \\
\left(-0.95^{* *}\right)\end{array}$} & $\begin{array}{l}1.06[0.74] \\
\left(0.86^{* * *}\right)\end{array}$ & \multicolumn{2}{|c|}{$\begin{array}{c}1.61[0.94] \\
\left(0.97^{* *}\right)\end{array}$} \\
\hline $\mathrm{CCW}^{11}, \mathrm{~kg}$ & $\begin{array}{l}0.62[0.74] \\
\left(0.86^{* * *}\right)\end{array}$ & $\begin{array}{c}0.05[0.67] \\
\left(0.81^{* *}\right)\end{array}$ & $\begin{array}{c}-0.67[0.72] \\
\left(-0.85^{* *}\right)\end{array}$ & \multicolumn{2}{|c|}{$\begin{array}{l}-2.28[0.85] \\
\left(-0.92^{* * *}\right)\end{array}$} & $\begin{array}{l}0.91[0.73] \\
\left(-0.85^{* * *}\right)\end{array}$ & \multicolumn{2}{|c|}{$\begin{array}{c}1.38[0.92] \\
\left(0.96^{* *}\right)\end{array}$} \\
\hline DR\%(cold) & $\begin{array}{c}0.01[0.66] \\
\left(0.24^{\mathrm{ns}}\right)\end{array}$ & $\begin{array}{c}-0.01[0.13] \\
\left(0.08^{\mathrm{ns}}\right)\end{array}$ & $\begin{array}{c}0.20[0.99] \\
\left(-0.99^{* * *}\right)\end{array}$ & \multicolumn{2}{|c|}{$\begin{array}{c}0.45[0.47] \\
\left(0.68^{*}\right)\end{array}$} & $\begin{array}{c}-0.11[0.16] \\
\left(-0.39^{\text {ns }}\right)\end{array}$ & \multicolumn{2}{|c|}{$\begin{array}{c}-0.34[0.82] \\
\left(-0.90^{* * *}\right)\end{array}$} \\
\hline $\mathrm{Fqw}$ (cold), $\mathrm{kg}$ & $\begin{array}{c}0.30[0.70] \\
\left(0.83^{* * *}\right)\end{array}$ & $\begin{array}{c}0.00[0.13] \\
\left(0.36^{\mathrm{ns}}\right)\end{array}$ & $\begin{array}{c}-0.05[0.39] \\
\left(-0.62^{*}\right)\end{array}$ & \multicolumn{2}{|c|}{$\begin{array}{c}-0.20[0.47] \\
\left(-0.68^{*}\right)\end{array}$} & $\begin{array}{c}0.02[0.04] \\
\left(0.21^{\mathrm{ns}}\right)\end{array}$ & \multicolumn{2}{|c|}{$\begin{array}{c}0.11[0.46] \\
\left(0.67^{*}\right)\end{array}$} \\
\hline $\mathrm{Hqw}$ (cold),kg & $\begin{array}{c}0.07[0.02] \\
\left(0.13^{\mathrm{ns}}\right)\end{array}$ & $\begin{array}{c}-0.76[0.99] \\
\left(-0.99^{* * *}\right)\end{array}$ & $\begin{array}{c}2.87[0.11] \\
\left(0.32^{* *}\right)\end{array}$ & \multicolumn{2}{|c|}{$\begin{array}{c}23.62[0.74] \\
\left(0.86^{* * *}\right)\end{array}$} & $\begin{array}{c}-11.40[0.92] \\
\left(-0.96^{* *}\right)\end{array}$ & \multicolumn{2}{|c|}{$\begin{array}{c}-10.26[0.42] \\
\left(0.64^{*}\right)\end{array}$} \\
\hline Bone,kg & $\begin{array}{c}0.06[0.72] \\
\left(0.84^{* * *}\right)\end{array}$ & $\begin{array}{c}0.01[0.69] \\
\left(0.83^{* * *}\right)\end{array}$ & $\begin{array}{c}-0.09[0.37] \\
\left(-0.60^{*}\right)\end{array}$ & \multicolumn{2}{|c|}{$\begin{array}{c}-0.45[0.91] \\
\left(-0.95^{* * *}\right)\end{array}$} & $\begin{array}{c}0.14[0.52] \\
\left(0.72^{* *}\right)\end{array}$ & \multicolumn{2}{|c|}{$\begin{array}{c}0.23[0.69] \\
\left(0.83^{* *}\right)\end{array}$} \\
\hline Meat,kg & $\begin{array}{c}0.04[0.72] \\
\left(0.84^{* * *}\right)\end{array}$ & $\begin{array}{c}0.02[0.18] \\
\quad(0.42)\end{array}$ & $\begin{array}{c}-0.66[0.99] \\
\left(-0.99^{* * *}\right)\end{array}$ & \multicolumn{2}{|c|}{$\begin{array}{c}-1.48[0.51] \\
\left(-0.71^{* *}\right)\end{array}$} & $\begin{array}{c}0.42[0.23] \\
\left(0.47^{\mathrm{ns}}\right)\end{array}$ & \multicolumn{2}{|c|}{$\begin{array}{c}1.11[0.85] \\
\left(0.92^{* *}\right)\end{array}$} \\
\hline Boneless meat $\%$ & $\begin{array}{c}0.02[0.15] \\
\left(0.38^{\text {ns }}\right)\end{array}$ & $\begin{array}{c}-0.01[0.83] \\
\left(-0.90^{* * *}\right)\end{array}$ & $\begin{array}{c}0.06[0.58] \\
\left(0.76^{* *}\right)\end{array}$ & \multicolumn{2}{|c|}{$\begin{array}{l}0.27[0.94] \\
\left(0.96^{* * *}\right)\end{array}$} & $\begin{array}{c}-0.11[0.83] \\
\left(-0.91^{* * *}\right)\end{array}$ & \multicolumn{2}{|c|}{$\begin{array}{c}-0.15[0.88] \\
\left(-0.93^{* * *}\right)\end{array}$} \\
\hline Fat,kg & $\begin{array}{c}0.08[0.79] \\
\left(0.89^{* * *}\right)\end{array}$ & $\begin{array}{c}0.01[0.64] \\
\left(0.80^{* *}\right)\end{array}$ & $\begin{array}{c}-0.18[0.59] \\
\left(-0.77^{* *}\right)\end{array}$ & \multicolumn{2}{|c|}{$\begin{array}{c}-0.62[0.69] \\
\left(-0.83^{* *}\right)\end{array}$} & $\begin{array}{c}0.28[0.78] \\
\left(0.88^{* * *}\right)\end{array}$ & \multicolumn{2}{|c|}{$\begin{array}{c}0.38[0.77] \\
\left(0.87^{* * *}\right)\end{array}$} \\
\hline \multicolumn{9}{|l|}{ Expensive cuts } \\
\hline Telbinco $^{12}, \mathrm{~kg}$ & $\begin{array}{c}0.01[0.44] \\
\left(0.66^{*}\right)\end{array}$ & $\begin{array}{c}0.00[0.32] \\
\left(0.56^{\mathrm{ns}}\right)\end{array}$ & $\begin{array}{c}-0.08[0.83] \\
\left(-0.91^{* * *}\right)\end{array}$ & $\begin{array}{r}-0.24[0 \\
(-0.86\end{array}$ & & $\begin{array}{c}0.06[0.26] \\
\left(0.50^{\mathrm{ns}}\right)\end{array}$ & & $0.89]$ \\
\hline Fillet $^{13}, \mathrm{~kg}$ & $\begin{array}{c}0.00[0.88] \\
\left(0.86^{* * *}\right)\end{array}$ & $\begin{array}{c}0.00[0.21] \\
\left(0.45^{\mathrm{ns}}\right)\end{array}$ & $\begin{array}{c}-0.09[0.92] \\
\left(-0.94^{* * *}\right)\end{array}$ & $\begin{array}{r}-0.24[0 \\
(-0.78\end{array}$ & & $\begin{array}{c}0.05[0.17] \\
\left(0.41^{\mathrm{ns}}\right)\end{array}$ & & $0.86]$ \\
\hline Roast beef ${ }^{14}, \mathrm{~kg}$ & $\begin{array}{c}0.03[0.71] \\
\left(0.82^{* * *}\right)\end{array}$ & $\begin{array}{c}0.01[0.54] \\
\left(0.73^{* *}\right)\end{array}$ & $\begin{array}{c}-0.09[0.85] \\
\left(-0.92^{* * *}\right)\end{array}$ & $\begin{array}{r}-0.28[0 \\
-0.90 \\
\end{array}$ & & $\begin{array}{c}0.10[0.58] \\
\left(0.76^{* *}\right)\end{array}$ & & $0.98]$ \\
\hline $\begin{array}{l}\text { [ ] Accuracy of simple re } \\
\text { Ns: non-significant* Sign } \\
\text { SW }^{1} \text {, slaughter weight; } \mathrm{H} \\
\text { hot carcass weight; } \mathrm{DR}^{8} \text {, } \\
\text { Telbianco }^{12} \text { and Roast be }\end{array}$ & $\begin{array}{l}\text { gression }{ }^{2} \text {. } \\
\text { ificant at } \mathrm{P}<0.05 \\
\mathrm{~W}^{2}, \text { height at with } \\
\text { dressing percenta } \\
\text { ef }^{13} \text {, cuts from rou }\end{array}$ & $\begin{array}{l}{ }^{*} \text { Significant a } \\
\text { r; } \mathrm{BL}^{3} \text {, body len } \\
; \mathrm{Fqw}^{9} ; \text { fore qu} \\
\text { d; Fillet }{ }^{14} \text {, cut } \mathrm{fr}\end{array}$ & $\begin{array}{l}\text { ) Simple ce } \\
0.01 . * * \text { Sig } \\
\text {; } \mathrm{DL}^{4} \text { diagona } \\
\text { r weight; Hqw } \\
\text { short loin. }\end{array}$ & $\begin{array}{l}\text { elation coe } \\
\text { icant at } \mathrm{P}< \\
\text { ngth } \\
\text { hind } \mathrm{HG} \text {, } \\
\text { huart }\end{array}$ & $\begin{array}{l}\text { icient. } \\
001 .\end{array}$ & $\begin{array}{l}\mathrm{RC}^{6}, \text { Round } \\
\mathrm{CW}^{11}, \text { cold ca }\end{array}$ & $\begin{array}{l}\text { cumf } \\
\text { ass w }\end{array}$ & $\mathrm{HCW}^{7}$ \\
\hline $\begin{array}{r}\text { Table 5. Multiple } \\
\text { by using bc }\end{array}$ & $\begin{array}{l}\text { ession eq } \\
\text { measurem }\end{array}$ & $\begin{array}{l}\text { ions to pr } \\
\text { s }\end{array}$ & arcass & of & & iesian cr & - & calv \\
\hline Dependent variable & & & Independe & variable & & & & $2(0 / 0)$ \\
\hline Depenuent variabre & intercept & $\mathbf{S W}^{1}$ & $\mathrm{HG}^{2}$ & $\mathbf{H W}^{3}$ & BL $^{4}$ & $\mathbf{D L}^{5}$ & $\mathbf{R C}^{6}$ & \\
\hline $\mathrm{HCW}^{7}$ & 842.632 & 0.212 & -4.098 & - & - & - & - & 94 \\
\hline $\mathrm{DR}^{8} \%$ (hot) & 255.27 & -0.10 & -1.14 & 0.32 & - & - & - & 91 \\
\hline Fqw $^{9}($ hot $)$ & 694.36 & - & -3.36 & - & - & - & - & 96 \\
\hline Hqw $^{10}$ (hot) & -108.44 & 0.22 & - & 1.01 & - & - & - & 96 \\
\hline $\mathbf{C} \mathbf{C W}^{11}$ & 2410.06 & 0.50 & -6.59 & -10.31 & - & - & - & 95 \\
\hline DR\%(cold) & 269.91 & -0.10 & -1.20 & 0.27 & - & - & - & 91 \\
\hline Fqw(cold) & 675.27 & - & -3.27 & - & - & - & - & 95 \\
\hline Hqw(cold) & -99.71 & 0.23 & - & 0.900 & - & - & - & 94 \\
\hline Bone & -66.28 & 0.06 & - & - & 0.91 & - & - & 99 \\
\hline Meat & -59.84 & 0.52 & - & - & - & - & - & 99 \\
\hline Boneless meat $\%$ & -78.76 & 0.09 & 0.65 & - & - & -0.35 & - & 99 \\
\hline $\begin{array}{l}\text { Fat } \\
\text { Prime cuts. }\end{array}$ & -175.28 & 0.25 & 0.92 & - & - & -0.71 & - & 99 \\
\hline$\frac{\text { Prime cuts: }}{\text { Telbinco }^{12}}$ & -37.36 & 0.02 & 0.19 & - & _- & - & & 88 \\
\hline Fillet $^{13}$ & 0.16 & 0.01 & - & -0.06 & 0.11 & - & - & 97 \\
\hline Roast beef $^{14}$ & -48.35 & 0.03 & 0.42 & - & - & - & 0.41 & 98 \\
\hline
\end{tabular}

$\mathrm{SW}^{1}$, slaughter weight; $\mathrm{HW}^{2}$, height at wither; $\mathrm{BL}^{3}$, body length; $\mathrm{DL}^{4}$ diagonal length ${ }^{5} ; \mathrm{HG}$, heart girth; $\mathrm{RC}^{6}, \mathrm{Round}$ circumference; $\mathrm{HCW}^{7}$, hot carcass weight; $\mathrm{DR}^{8}$, dressing percentage; $\mathrm{Fqw}^{9}$; fore quarter weight; $\mathrm{Hqw}^{10}$, hind quarter weight ; $\mathrm{CCW}^{11}$, cold carcass weight; Telbianco ${ }^{12}$ and Roast beef ${ }^{13}$, cuts from round; Fillet ${ }^{14}$, cut from short loin. 
Table 6. Multiple regression equations to predict carcass traits of fattening buffalo male calves by using body measurements

\begin{tabular}{|c|c|c|c|c|c|c|c|c|}
\hline \multirow[t]{2}{*}{ Dependent variable } & \multicolumn{7}{|c|}{ Independent variable } & \multirow[t]{2}{*}{$\mathbf{R}^{2}(\%)$} \\
\hline & Intercept & $\mathbf{S W}^{1}$ & $\mathrm{HG}^{2}$ & $\mathbf{H W}^{3}$ & $\mathbf{B L}^{4}$ & $\mathbf{D L}^{5}$ & $\mathbf{R C}^{6}$ & \\
\hline $\mathrm{HCW}^{7}$ & -126.72 & 0.64 & - & - & 1.10 & - & - & 99 \\
\hline$D^{8} \%$ (hot) & 37.57 & - & - & - & 0.21 & - & - & 98 \\
\hline Fqw $^{9}($ hot $)$ & 145.27 & - & - & - & -0.22 & - & - & 99 \\
\hline $\mathrm{Hqw}^{10}$ (hot) & -108.44 & 0.22 & - & 1.01 & - & - & - & 94 \\
\hline $\mathbf{C} \mathbf{C} \mathbf{W}^{11}$ & 95.38 & - & - & - & - & - & 1.38 & 92 \\
\hline DR\%(cold) & 35.82 & - & - & - & 0.20 & - & - & 99 \\
\hline Fqw(cold) & 183.19 & - & 0.17 & - & - & -0.54 & - & 97 \\
\hline Hqw(cold) & 206.4 & - & - & -0.76 & - & - & - & 99 \\
\hline Bone & 83.77 & - & - & - & - & -0.45 & - & 99 \\
\hline Meat & 179.36 & - & - & - & -0.66 & - & - & 99 \\
\hline Boneless meat\% & 6.69 & - & - & - & - & 0.27 & - & 94 \\
\hline Fat & -98.26 & 0.20 & - & - & 0.40 & - & - & 96 \\
\hline \multicolumn{9}{|l|}{ Expensive cuts: } \\
\hline Telbinco & -8.47 & - & - & - & - & - & 0.15 & 89 \\
\hline Fillet & 11.35 & - & - & - & -0.09 & - & - & 90 \\
\hline Roast beef & -6.07 & - & - & - & - & - & 0.17 & 98 \\
\hline
\end{tabular}

$\mathrm{SW}^{1}$, slaughter weight; $\mathrm{HW}^{2}$, height at wither; $\mathrm{BL}^{3}$, body length; $\mathrm{DL}^{4}$ diagonal length ${ }^{5} ; \mathrm{HG}$, heart girth; $\mathrm{RC}^{6}$, Round circumference; $\mathrm{HCW}^{7}$, hot carcass weight; $\mathrm{DR}^{8}$, dressing percentage; $\mathrm{Fqw}^{9}$; fore quarter weight; $\mathrm{Hqw}^{10}$, hind quarter weight; $\mathrm{CCW}^{11}$, cold carcass weight; Telbianco ${ }^{12}$ and Roast beef ${ }^{13}$, cuts from round; Fillet ${ }^{14}$, cut from short loin.

\section{CONCLUSION}

The slaughter weights of both species studied were positively and significantly correlated with most carcass traits. In Friesian crossbred calves, heart girth (HG) was significantly correlated with most of carcass traits but in buffalo calves round circumference (RC) is considered the first body measure which is positively and strongly associated with most carcass traits and followed by BL. Therefore, the slaughter weight, heart girth, round circumference and body length, are most important indicators to predict the carcass traits in both species.

\section{REFERENCES}

Afolayan R.A., M.P.B. Deland, D.L. Rutley, C.D.K..Bottema, A.L. Ewers, R.W. Ponzoni and W.S. Pitchford, 2002. Prediction of carcass meat, fat and bone yield across diverse cattle genotypes using live-animal measurements. Anim. Prod. Aust. 24, 13-16.

Afolayan R.A., W.S. Pitchford and C.D.K. Bottema, 2001. Genetic effects on shape and fatness of calves from diverse crosses.Proc. Aust. Assoc. Anim. Breed. Gen.14, 481-4.

Ayyat M.S., I.F. Marai and H.M. Farghaly, 1997.A trial to grade Egyptian cattle for beef production according to fleshing and its associated traits. International Conference on Animal, Poultry and Rabbit production and health. Zagazig University. 9, 2-4.

Bonvillani A., F Pena, G. de Gea, G. Gomez, A. Petryna and J. Perea, 2010. Carcass characteristics of Criollo Cordobes kid goat under an extensive management system: Effect of gender and live weight at slaughter .Meat science. Nov,86(3):651-9 .

FaizurRahman M.D., 2007. Prediction of carcass weight from the body characteristics of Black Bengal Goats.International Journal of Agriculture \& Biology.3, 431- 434

Fisher, A. V., 1976. Live animal measurements as a means of evaluating animals in beef production experiments. In seminar on criteria and methods for assessment of carcass and meat characteristics in beef production Experiments, zeist, Holland.

Gilbert, R. P.; Bailey, D.R.C. and Shannon, N. H.(1993). Body Dimensions and Carcass Measurements of Cattle Selected for Post weaning Gain Fed Two Different Diets. J. Anim. Sci. 71, 1688-1698.

Kohli, M. L., A. C. Cook and W. M. Dawson.(1951). Relations between some body measurements and certain performance characters in Milking Shorthorn steers. J. Anim. Sci. 10,352-364.

NRC, National Research Council (2001).Nutrient Requirements of Beef Cattle.National Academy Press, Washington, DC.

Ogah D. M1., I. S. Musa and N. D. Yusuf.(2012). Prediction of carcass weight from body measurements in West African Dwarf goat using Canonical Correlation Analysis.Egyptian Journal of Sheep and Goat Sciences. 8, 75-79.

Rahman F. M. D. (2007). Prediction of carcass weight from the body characteristics of black Bengal goat Inter. J. of Agric. and Biol.3,431434.

Rashad, A.M.A. (2009). Studies on some economic traits in mail Egyptian buffalo calves from suckling to marketing. Master of Science in Agricultural Sciences in Animal production.Alexandria University. Egypt. 
Sadek, R. R., Morsy, M. A., Nigm, A. A., Ibrahim, M. A. M. and Sabry, A. M. (1993). The effect of grading up Baladi cattle with Friesian on meat production performance. Egyptian J. Anim. Prod. 30,143-160.

SAS. (2004). SAS User's Guide:Basico SAS Ins., Carry. N.C.
Shelton M.,G. C. Smith and F. Orts (1977). Predicting carcass cutability of Rambonillet rams using live animal traits. J Anim. Sc. 44, 333-337.

Yao, T. S.; Dawson, W. M. and Cook, A. C. (1953). Relationships between Meat Production Characters and Body Measurements in Beef and Milking Shorthorn Steers. J. Anim. Sci. 12, 775786.

التتبؤ بصفات الأبيحة لأكور العجول الفريزيان الخليطة والجاموس المسمنة باستخدام قياسات الجسم

داليا قاسم عبدالمنعم الحديني، محمدجمال الدين عبداللطيف، أحمد الطاهر مهدي

قسم الإنتاج الحيواني، كلية/لزراعة الثاطبي، جامعة الاسكندرية

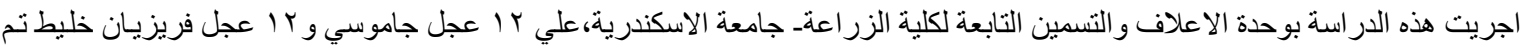

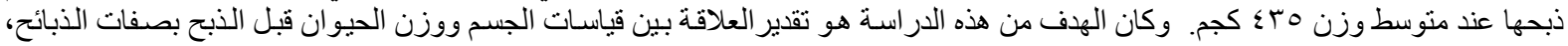

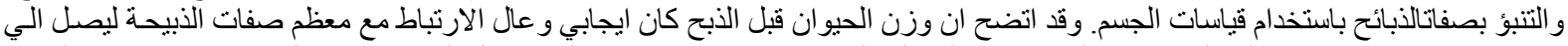

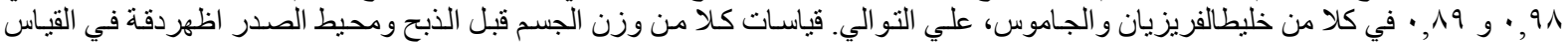

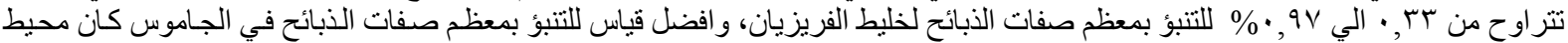

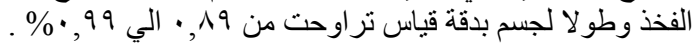

